\title{
A novel device for removal of the retained intrauterine device
}

\author{
Shamprasad P Konamme, Barry J Auld, Jamal Zaidi
}

\begin{abstract}
Objective To evaluate the efficacy and acceptability of the Macaluso stent removal forceps for removal of retained intrauterine devices (IUDs) under direct vision using a hysteroscope in an outpatient setting.

Methods Nineteen consecutive patients with a retained IUD who had been referred from primary care to the gynaecologists at the Conquest Hospital, Hastings, UK were included in the study. Attempts were made to remove the IUDs under direct vision using the Gynecare Versascope ${ }^{\circledR}$ and Macaluso forceps.
\end{abstract}

Results The procedure was successful in 18/19 (94.7\%) patients. The patients tolerated the procedure well and none of them complained of severe pain.

Conclusion This device is simple, safe, effective and acceptable for the removal of IUDs with lost threads.

J Fam Plann Reprod Health Care 2006; 32(2): 87-88 (Accepted 4 January 2006)

\section{Key message points}

- Various instruments have been used for removal of retained intrauterine devices (IUDs) with variable success rates reported.

- Hysteroscopy is an effective technique under either general or local anaesthesia.

- The Macaluso stent removal forceps is a useful device for hysteroscopic IUD removal.

\section{Introduction}

Lost devices account for approximately $9 \%$ of follow-up visits from women who have been fitted with an intrauterine contraceptive device (IUD). ${ }^{1}$ Management consists of attempted thread retrieval with forceps, thread retrievers or hooked instruments, usually in the outpatient setting, but with varying success rates. ${ }^{1}$ Bounds et al., in a randomised study evaluating three different thread retrievers, found that in approximately $40 \%$ of all patients the threads were retrieved with Spencer Wells forceps alone and in a further $40 \%$ of cases with disposable plastic retrievers. ${ }^{2}$ Cervical dilatation and exploration of the uterus, with or without hysteroscopy, under general anaesthesia has been the only alternative for these patients. ${ }^{1,2}$ Hysteroscopy and removal of an IUD have been described previously. ${ }^{3-5}$ However, no forceps has been described in the literature that is suitable for removing the IUD under direct vision via the hysteroscope.

Department of Obstetrics and Gynaecology, Conquest Hospital, St Leonards on Sea, UK

Shamprasad P Konamme, MRCOG, Specialist Registrar in Obstetrics and Gynaecology

Barry J Auld, FRCOG, Consultant in Obstetrics and Gynaecology Jamal Zaidi, MD, FRCOG, Consultant in Obstetrics and Gynaecology

Correspondence to: Dr S P Konamme, Department of Obstetrics and Gynaecology, Royal Victoria Infirmary, Leazes Wing, Queen Victoria Road, Newcastle-upon-Tyne NE1 4LP, UK.

E-mail: skonamme@aol.com
We used our existing one-stop hysteroscopy clinic for the diagnosis and removal of IUDs with lost threads. Unavailability of suitable forceps for this procedure and observation of our urology colleagues encouraged us to try Macaluso stent removal forceps, in combination with the Gynecare Versascope ${ }^{\circledR}$. The Macaluso forceps has been used previously specifically for the removal of ureteric stents. The forceps has the particular attributes of being both very small gauge ( $3 \mathrm{Fr}$ ) but with an extrudable grasping mechanism in the form of three hooked prongs, which exert a very good grip on a narrow filamentous structure. The prongs of the Macaluso forceps will catch the coil threads or coil eyelet with ease as long as the tip of the forceps is near the threads. The Macaluso forceps comes in a single-use disposable pack and costs around $£ 18.00$.

This observational study evaluates the advantages and disadvantages of using this device for the removal of IUDs with lost threads.

\section{Methods}

This was a retrospective observational study. Nineteen consecutive patients who had been referred from primary care to the Gynaecology Department at the Conquest Hospital, Hastings, UK with lost IUD threads were included in the study. The technique was evaluated initially in two patients under general anaesthesia who were on the waiting list for dilatation and exploration for lost IUD threads. This was to ensure that the proposed instrumentation performed adequately before subjecting patients to a local anaesthetic procedure. These two cases were excluded from the study. The next 19 patients were seen in the outpatient hysteroscopy clinic and underwent the procedure under local anaesthesia. The patients were aged between 25 and 53 years, with the period of IUD retention varying from 2 to 14 years from the time of insertion until referral to the hospital. The various retained IUDs were as follows: Copper $\mathrm{T}^{\circledR}(8)$, Mirena ${ }^{\circledR}$ IUS (7), Copper $7^{\circledR}(2)$, Lippes loop ${ }^{\circledR}(1)$ and Multiload ${ }^{\circledR}$ Copper (1).

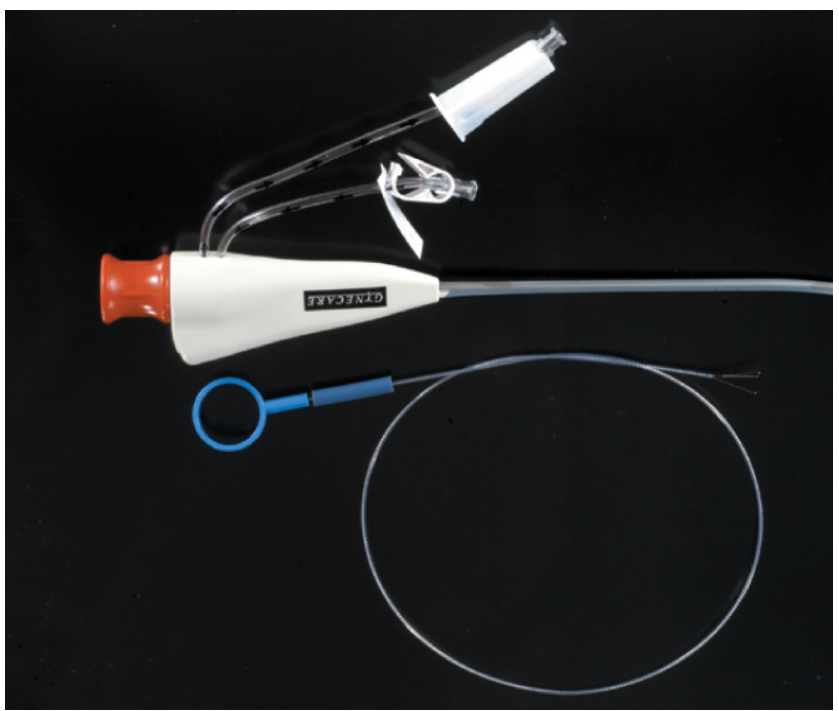

Figure 1 Gynecare Versascope ${ }^{\circledR}$ and Macaluso forceps 


\section{ARTICLE}

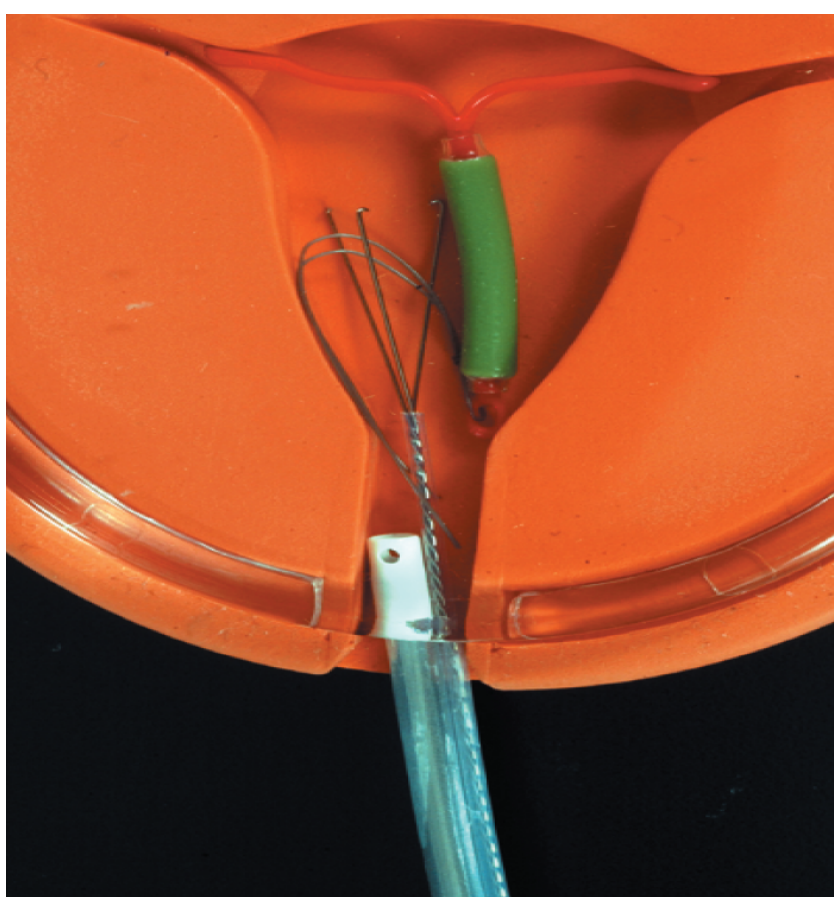

Figure 2 Grasping the intrauterine device threads

All patients underwent a transvaginal ultrasound scan in the 'one-stop' outpatient hysteroscopy clinic. After history taking, clinical examination and a transvaginal ultrasound scan, hysteroscopy was performed under aseptic conditions. A Cusco speculum was introduced to visualise the cervix. Local anaesthetic paracervical block (Citanest ${ }^{\circledR} 2.5 \mathrm{ml}$ ) was given using a dental syringe. We used the Gynecare Versascope rigid $3 \mathrm{~mm}$ hysteroscope (Gynecare, Edinburgh, UK) for the hysteroscopy (Figure $1)$. Once the coil was located in the uterine cavity, a Macaluso stent removal forceps (Microvasive, Boston Scientific Ltd, St Albans, UK) was introduced through the operating channel of the hysteroscope (Figure 2). The Macaluso forceps has three retractable prongs. The prongs open and can then be used to grasp either the coil threads (Figure 2) or the eye of the coil. The forceps is then removed with the coil under vision together with the hysteroscope (Figure 3). The uterine cavity is visualised once again with the hysteroscope to rule out any other pathology. The duration of the procedure ranged between 5 and 10 minutes.

\section{Results}

The procedure was successful in 18/19 cases. Threads were totally absent from the stem of the IUD in four cases including the one Multiload device. We failed to remove only one IUD, namely the Multiload device without thread. The Multiload had no eye at the bottom of the shaft and therefore it was difficult to grip with the forceps. This coil was also embedded within the uterine cavity. This patient required a general anaesthetic procedure in which the coil was removed blindly after repeated attempts using a long Spencer Wells forceps. We succeeded in the removal of eight Copper T, seven Mirena IUS, two Copper 7 and one Lippes loop. Our overall success rate for the procedure was $94.7 \%$.

All the patients who had their IUD removed under local anaesthetic hysteroscopy appeared to tolerate the procedure well. None of the patients were observed to complain of any severe pain and there were no complications.

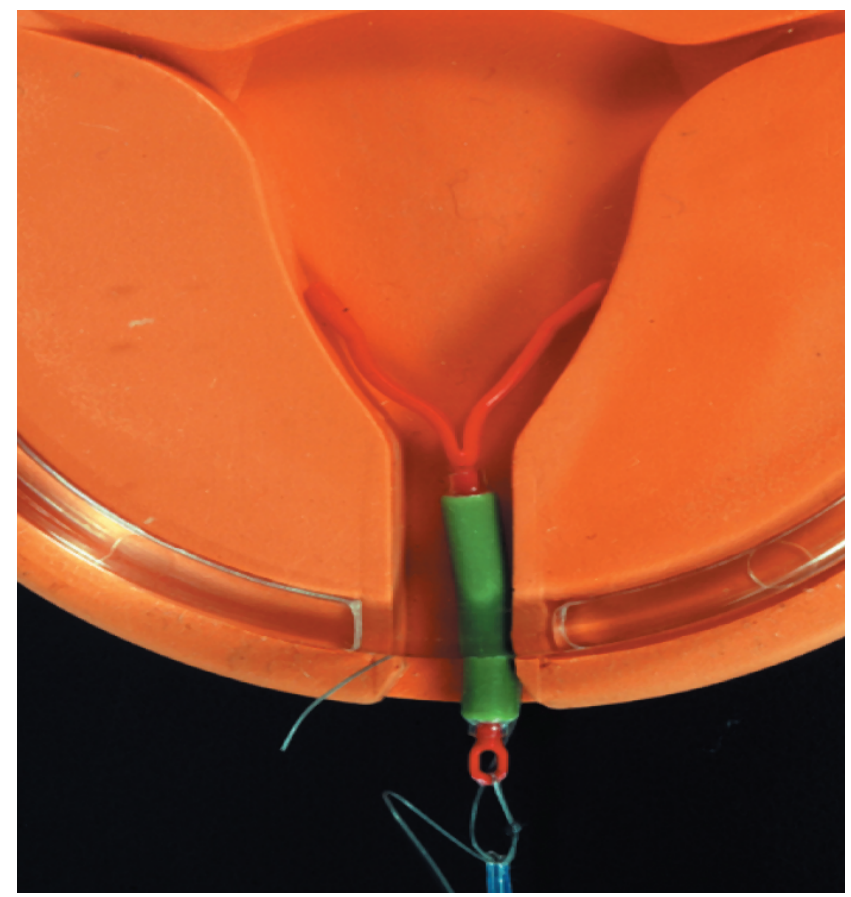

Figure 3 Retrieving the intrauterine device

\section{Discussion}

The lost thread of an IUD can be retrieved using a simple forceps, brush, thread retriever device or hook in the primary care setting. ${ }^{2}$ If this fails then patients are referred to secondary care to identify and/or remove the device. We agree with Trivedi et al. that blind manipulation with artery forceps, hooks and clamps for IUD retrieval may be potentially dangerous, producing cervical or uterine injuries. ${ }^{5}$ Using a hysteroscope to locate and remove IUDs under general anaesthesia is a well-established technique. The risk of uterine perforation is minimal when the procedure is performed under direct vision..$^{5}$

The present results demonstrate that Macaluso stent removal forceps with Versascope hysteroscopy for the removal of retained IUDs is a simple, safe, effective and acceptable technique. We recommend this procedure as a first-line option for the removal of a retained IUD in a secondary care setting. We accept that the cost of the procedure may be higher than the blind manipulation techniques; however, the added safety and efficacy outweigh the cost differential.

Our experience suggests that the Macaluso forceps is a useful device for the specific purpose of hysteroscopic IUD removal.

Statements on funding and competing interests

Funding. None identified.

Competing interests. None identified.

References

1 Chamberlain G. The missing tail of an intrauterine device. Br J Fam Plann 1982; 8: 50-51.

2 Bounds W, Hutt S, Kubba A, Cooper K, Guillebaud J, Newman GB. Randomised comparative study in 217 women of three disposable plastic IUCD thread retrievers. Br J Obstet Gynaecol. 1992; 99: 915-919.

3 Valle RF, Sciarra JJ, Freeman WD. Hysteroscopic removal of intrauterine devices with missing filaments. Obstet Gynecol 1977; 49: 55.

4 Gupta I, Devi PK, Gupta AN. Hysteroscopic removal of intrauterine contraceptive devices with missing threads. Ind J Med Res 1977; 65: 661-663.

5 Trivedi SS, Goel M, Jain S. Hysteroscopic management of intrauterine devices with lost stings. Br J Fam Plann 2000; 26: 229-230. 